\title{
The influence mechanism of UAV group on the detection performance of air defense radar
}

\author{
Zhiyu Zhou $^{\text {a) }}$, Bin Rao, Xiaoxia Xie \\ State Key Laboratory of Complex Electromagnetic Environment Effects on Electronics and Information System, National University of Defense Technology, \\ Changsha 410073, People's Republic of China \\ a) zhouzhiyu16a@nudt.edu.cn
}

\begin{abstract}
This paper investigates the detection performance of densely unmanned aerial vehicle (UAV) group on the air defense radar. The keystone is that multiple UAVs frequently fly in adjacent range resolution cell and usually locate in the same beam cell that inevitably has influence on the angle resolution capability of tradditional radar. Firstly, the monopulse angle estimation of unresolved targets is presented, this is inherently the classic dual source jamming model. Sencondly, to show the merging phenomenon of angle estimation, the probabilty density distribution related to multiple observations and different Swerling targets is given. Finally, a simulation involving parallel, cross and random flight trajectories on the detection performance of radar is given. It is found that when the number of UAV increases, or the spatial distribution of UAVs becomes denser, the probability of measurement merging increases. It is also found that if the beam width of radar increases, or the bandwidth of the radar signal decreases, the probability of measurement merging increases.
\end{abstract}

Keyword: monopulse; angle estimation; unresolved targetS; UAV; detection performance

\section{INTRODUCTION}

The monopulse method is currently widely used in radar angle estimation. However, when multiple targets fall within the same or adjacent range resolution cells, the conventional monopulse radar system cannot distinguish between multiple targets [1]. As a result, the angle estimation obtained by the conventional signal processing method is actually the energy centroid of multiple targets, rather than the true angles of each target. On the other hand, especially in recent years, rapid development of UAV technology has raised much attention. The use of UAVs for cooperative electronic countermeasure (including reconnaissance, jamming, barrage, anti-radiation, etc.) against radar is also a major application for military UAVs. However, the actual influence of densely UAVs on air defense radar still needs to be investigated. Multiple UAVs frequently fly in adjacent range resolution cell and usually locate in the same beam cell that inevitably has influence on the range, angle, and velocity resolution capabilities of traditional radar. Especially, the angle merging should be highlighted, as the radar might arrive an erroneous estimation number of target if the single angle estimation is inherently consisted of multiple targets. [3]

This paper first theoretically analyzes the influence of two simple point targets located in the same range resolution cell on the monopulse radar angle measurement. Then the studying of the influence of two simple point targets with Swerling target model of type 3 on the radar is presented. Finally, simulation results including parallel, cross, and random flight trajectories were given to verify the impacts of multiple UAVs on radar.

\section{MONOPULSE ANGLE ESTIMATION OF UNRESOLVABLE TARGETS IN ONE PULSE}

It is acknowledged in [1] that:

$$
\frac{d}{S}=k_{m} \theta
$$

where $s, d$ is the sum and difference voltage respectively, $\theta$ is the angle off axis in that coordinate expressed in beam widths, and $k_{m}$ is the slope of the monopulse response curve versus angle in the same coordinate. 
As in [1] the indicated angle is

$$
\theta_{i}=\frac{1}{k_{m}} \frac{d}{s}=\frac{1}{k_{m}} \frac{d_{a}+d_{b}}{s_{a}+s_{b}}=\frac{\theta_{a} s_{a}+\theta_{b} s_{b}}{s_{a}+s_{b}}
$$

Where the sum and difference voltage of the first target is respectively $s_{a}, d_{a}$, and the sum and difference voltage of the second target is respectively $s_{b}, d_{b}$.

Let

\section{Where}

$$
s_{b} / s_{a}=p e^{j \phi}
$$

$p=g r$ = amplitude ratio of sum-signal returns from the two targets;

$g$ = ratio of antenna sum-pattern voltage gains in the directions of the two targets;

$r$ = ratio of backscatter voltage coefficients of the two targets (the square root of the ratio of their radar cross sections);

$\phi=$ relative phase of the sum-signal returns from the two targets.

Then it can be obtained:

$$
\theta_{i}=\theta_{\text {mid }}-\frac{\Delta \theta}{2} \frac{1+p e^{j \phi}}{1+p e^{j \phi}}
$$

where $\theta_{\text {mid }}=\frac{\theta_{a}+\theta_{b}}{2}, \Delta \theta=\theta_{a}-\theta_{b}$.

And the real terms of $\theta_{i}$ can be expressed in this form:

$$
\operatorname{Re}\left(\theta_{i}\right)=\theta_{\text {mid }}-\frac{\Delta \theta}{2} \frac{1-p^{2}}{1+2 p \cos \phi+p^{2}}
$$

Figure 1 shows the real terms of $\theta_{\text {in }}$, which is the real part of the normalized indicator, it's a function of $\phi$ at different values of $p$, calculated by (5), and normalized by (6).

$$
\theta_{i n}=\frac{\theta_{i}-\theta_{m i d}}{-\Delta \theta}=\frac{1}{2} \frac{1-p e^{j \phi}}{1+p e^{j \phi}}
$$

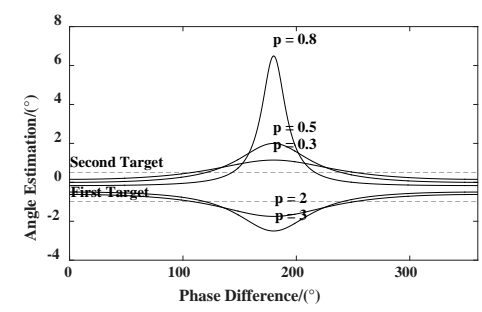

FIGURE 1. The real part of the normalized indicator.

Figure 1 show the monopulse estimation result using a single pulse when the amplitude ratio $r$ takes different values with the phase difference $\phi$ as a variable. For illustration, only 5 curves are shown in the figure. As can be seen from the figure, the angle estimation of which the phase difference near $180^{\circ}$ deviates from the centroid. 


\section{MONOPULSE ANGLE ESTIMATION FOR MULTIPLE OBSERVATIONS OF UNRESOLVABLE TARGETS}

Monopulse radars usually use multiple observations to estimate the direction of arrival of a target (DOA). For fluctuating targets, Mosca has proposed a maximum likelihood (ML) DOA estimation method under I times of independent measurements [2].

$$
\hat{\eta}_{M L} \approx \operatorname{Re}\left\{\frac{\sum_{i=1}^{I} s_{i}^{H} d_{i}}{\sum_{i=1}^{I}\left|s_{i}\right|^{2}}\right\}
$$

where $\eta_{M L}$ is the ML expression for DOA estimator, $s_{i}, d_{i}$ is the $i^{\text {th }}$ sum and difference voltage respectively.

The following experiment discusses the different monopulse angle estimation simulation results about unresolvable targets under slow fluctuations and fast fluctuations. A total of 10,000 times Monte Carlo experiments are used for two unresolvable Swerling targets of type 2 and two unresolvable Swerling targets of type 1, respectively. In each Monte Carlo experiment, the ML monopulse angle estimation with 20 independent observations was used.

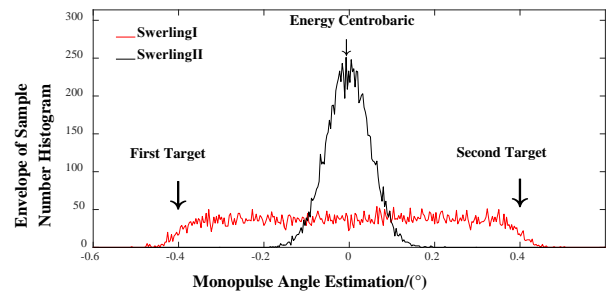

FIGURE 2. Statistical results of ML angle estimation for two unresolvable targets.

\section{MONOPULSE ANGLE ESTIMATION OF UAVS IN SPECIFIC SCENES}

\section{Scene One}

Scene 1 is set as two UAV targets flying along a trajectory as shown in Figure 3 in a uniform linear motion and approaching the radar. The parameters are set to: the beam width of radar is $2^{\circ}$ The RCS of two UAV targets are subjected to the Swerling III target model. The radar signal bandwidth is $10 \mathrm{MHz}$, the pulse repetition interval is $6 \mathrm{~ms}$, and the sampling frequency is $50 \mathrm{MHz}$. The amplitude ratio of the two target echo signals is 1 and the phases of echo signals are uniformly distributed.

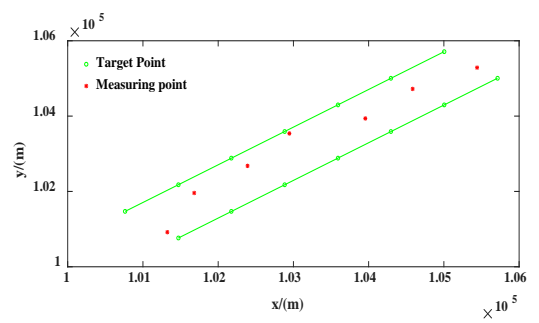

FIGURE 3. Measuring points of two parallel moving target track.

Figure 3 depicts the simulation results, the red points are the measuring points, the green ones are the target points, and it's obvious that in the same distance resolution unit, a conventional monopulse radar cannot use sumdifference beam measurements and constant false alarm rate (CFAR) detecting to distinguish two fluctuating targets. The result above is one random point between the two actual target's spatial ranges at the current moment but not the energy centroid between two targets because of the random phase difference and signal noises. 
In this scene, concentrations are also to be paid on the changes of the detection performance due to differences in radar signal bandwidth and radar beam width. First, radar beam width is fixed to $0.5^{\circ}$ and changing the radar signal bandwidths to observe the radar detection performance.

TABLE 1. Comparison of different radar signal bandwidths in scene 1.

\begin{tabular}{ccc}
\hline B/(MHz) & MP & DP \\
\hline 0.5 & 200 & 178 \\
0.45 & 200 & 175 \\
0.4 & 200 & 168 \\
0.35 & 200 & 148 \\
0.3 & 200 & 128 \\
\hline
\end{tabular}

where B represents the radar signal bandwidth, MP represents the number of measuring points, and DP represents the number of detected points in the detection.

From Table 1, it can be seen that when the radar beam width is fixed, there is measurement performance degradation in scene 1 as with the radar bandwidth reduction. This is because when the band width of the radar signal decreases, the size of the radar distance resolution cell increases, and the number of two measuring points in the same distance resolution unit increases, when the radar beam width is constant, the probability of measurement merging increases.

Next, radar signal band width is fixed to $0.5 \mathrm{MHz}$ and changing the radar beam widths to observe the radar detection performance.

TABLE 2. Comparison of different radar beam widths in scene 1.

\begin{tabular}{ccc}
\hline Theta $/\left(^{\circ}\right)$ & MP & DP \\
\hline 0.5 & 200 & 178 \\
0.4 & 200 & 178 \\
0.3 & 200 & 179 \\
0.2 & 200 & 184 \\
0.1 & 200 & 192 \\
\hline
\end{tabular}

It can be seen in Table 2 that when the radar signal bandwidth is fixed, the detection performance of the radar increases as the decrease of its beam width. This is because when the radar signal bandwidth is fixed, the decrease of the beam width will cause the number of measuring points falling in the same beam to decrease. As a result, the probability of measurement merging in radar detection is reduced.

\section{Scene Two}

Scene 2 is set as two UAV targets along the trajectory as shown in Figure 4, and two targets fly in the direction close to the radar in the uniform linear motion. The parameter settings are the same as that in the scene 1 .

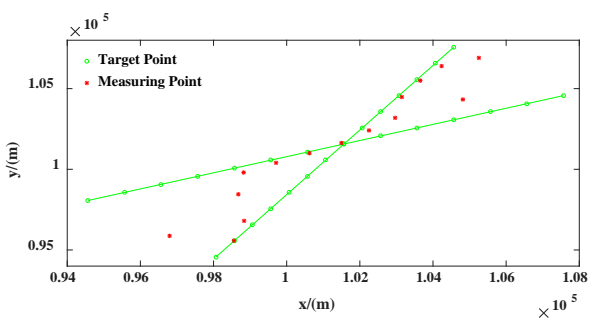

FIGURE 4. Measuring points of two crossing moving target track.

The simulation result of scene 2 is shown in Figure 4. Similarly, the result shows that the conventional monopulse radar cannot distinguish between two targets in the same distance cell, and the two measuring points obtained in some frames of the motion process in Figure 4 are due to the selection of the threshold factor in CFAR detection, resulting in two measuring points over a small distance range. It can be seen from Figure 4 that the impact of two moving targets on radar ranging is not obvious, but mainly affects the angle measurement performance of conventional monopulse radars. 
Similarly, fixing the radar beam width to $2^{\circ}$ and the radar signal bandwidth to $2 \mathrm{MHz}$ respectively in scene 2 and observing the change in another measurement parameter to judge the influence on the radar detection performance, the results are Table 3 and Table 4.

TABLE 3. Comparison of different radar signal bandwidths in scene 2.

\begin{tabular}{ccc}
\hline $\mathbf{B} /(\mathbf{M H z})$ & MP & DP \\
\hline 2 & 200 & 189 \\
1.8 & 200 & 188 \\
1.6 & 200 & 186 \\
1.4 & 200 & 184 \\
1.2 & 200 & 184 \\
\hline
\end{tabular}

TABLE 4. Comparison of different radar beam widths in scene 2.

\begin{tabular}{ccc}
\hline Theta $/\left({ }^{\circ}\right)$ & MP & DP \\
\hline 2 & 200 & 189 \\
1.6 & 200 & 191 \\
1.2 & 200 & 192 \\
0.8 & 200 & 193 \\
0.4 & 200 & 197 \\
\hline
\end{tabular}

The results of Table 3 and Table 4 are basically consistent with those in scene 1 . Since the flight trajectory of scene 2 is more complex than scene 1, the radar signal bandwidth B and beam width Theta are chosen differently from scene 1 .

\section{Scene Three}

The trajectory of scene 3 is shown in Figure 5. It is a scene in which a certain range of 10 UAVs fly to the target located in $(500 \mathrm{~km}, 0)$ at a speed of $240 \mathrm{~m} / \mathrm{s}$ after receiving an attack command, and the simulation time is $100 \mathrm{~s}$. The horizontal and vertical coordinates of the initial position of the ten targets obey the uniform distribution from $50 \mathrm{~km}$ to $100 \mathrm{~km}$, respectively, and the initial velocity direction is set to be uniformly distributed within $(-\pi, \pi)$, and then the angle is gradually adjusted during the motion to fly at a uniform linear velocity to the target point.

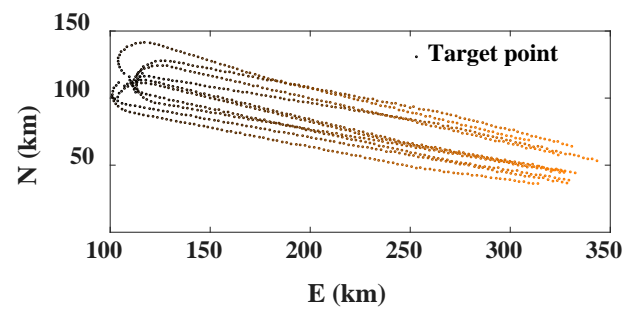

FIGURE 5. The trajectory of scene 3.

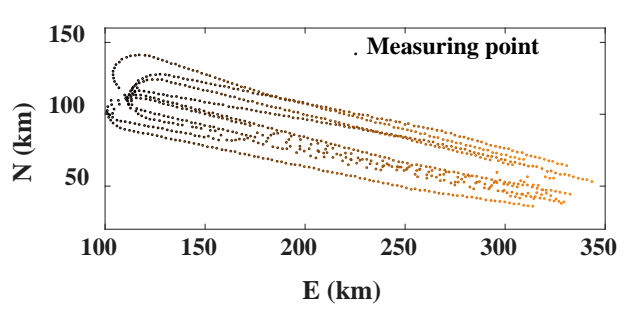

FIGURE 6. Measuring results of scene 3.

From the comparison of Figure 5 and Figure 6, it can be seen that when multiple targets located in the same beam at the same time are distributed in the same distance resolution cell, the phenomena of measurement merging will obviously occur. Therefore, compared with the previous two scenes, when the number of UAVs is larger and the distribution is relatively intensive, the number of measurement merging points increases. 


\section{CONCLUSION}

This paper firstly analyzes classic dual source model measurements using the monopulse radar in theory. It is found that the conventional monopulse radar cannot distinguish between two targets in adjacent range resolution cell and usually located in the same beam cell. The results of simulation involving single and multiple observations prove the validity of the theoretical analysis. The results of the simulation involving parallel cross and random flight trajectories of UAVs on the detection performance of radar also demonstrate that the conventional monopulse radar system cannot distinguish between multiple targets in adjacent range resolution cell and usually locate in the same beam cell.

By observing the measurement merging points, when the signal bandwidth of radar is reduced and the beam width is increased, the detection performances of the monopulse radar will suffer a decline. However, when those two parameters reach a certain range, the influence on the detection performances of the monopulse radar is relatively small. In addition, this paper only discusses the influence of two factors on the monopulse radar detection performances, which are, the distribution and trajectories of UAVs themselves. If the impact of external clutter and the cooperative electronic countermeasure from UAVs on the monopulse radar is taken into account, it's believed that the probability of measurement merging will be even higher. Further research will be conducted on those two factors.

\section{ACKNOWLEDGMENTS}

This work was supported by the National Scientific Fund of Chinese (No. 61201335 and No. 61571451) and the Fund of State Key Laboratory of Complex Electromagnetic Environment Effects on Electronics Information System (No. CEMEE2018K0303B).

\section{REFERENCES}

1. S.M. Sherman and D. K. Barton, Monopulse Principles and Techniques, $2^{\text {nd }}$ ed.; Artech House: 685 Canton Street Norwood, MA 02062, USA, 2011; pp.190-199.

2. Mosca E., Angle estimation in amplitude comparison monopulse system. IEEE Trans. on Aerospace and Electronic Systems, 1969,5(2):205-212

3. Zhao Yi-nan, Yao Jian, Li Feng-cong, Angle estimation for slow-fluctuation unresolvable targets with monopulse radar. Systems Engineering and Electronics, 2010,32(10):2021-2024 